\title{
Mesons in the medium - experimental probes for chiral symmetry restoration?
}

\author{
Mariana Nanova*t \\ II. Phys. Institut, JLU - Giessen, Germany \\ E-mail: Mariana.Nanova@exp2.physik.uni-giessen.de
}

The in-medium modifications of hadron properties have been identified as one of the key problems in understanding the non-perturbative sector of QCD. Several theoretical papers discuss the possibility of a partial restoration of chiral symmetry in a strongly interacting environment. However, is it possible to find experimental evidence for partial symmetry restoration by studying the in-medium behaviour of mesons, and the meson-nucleus interaction. Is this interaction sufficiently strong to allow even the formation of mesic states only bound by the strong interaction? The answers can be given by studying the meson-nucleus complex potential. The experimental approaches to deduce this potential and the experimental results from CBELSA/TAPS are presented and discussed. Data taken on a $\mathrm{C}$ and $\mathrm{Nb}$ target at the Crystal Barrel (CB)/TAPS detector system at the ELSA accelerator facility in Bonn have been analyzed to deduce the $\eta^{\prime}$ - and $\omega$-nucleus potential. The data for both mesons are consistent with a weakly attractive potential. The formation and population of $\omega$-nucleus and $\eta^{\prime}$-nucleus bound states is discussed. In case of the $\omega$ meson the in-medium width is found to be larger than the potential depth which hampers a successful experimental identification of $\omega$-mesic states. The relatively small in-medium width of the $\eta^{\prime}$ meson encourages ongoing experiments to search for $\eta^{\prime}$-nucleus bound states.

55th International Winter Meeting on Nuclear Physics

23-27 January, 2017

Bormio, Italy

\footnotetext{
*Speaker.

${ }^{\dagger}$ on behalf of the CBELSA/TAPS Collaboration
} 


\section{Introduction}

The origin of hadron masses and predicted in-medium modifications of hadron properties are key issues in QCD in the strong coupling regime. Widespread theoretical and experimental studies focus on the question whether the hadron properties change when the hadrons are embedded in a strongly interacting environment $[1,2,3,4]$. Mass shifts and/or in-medium broadening as well as more complex structures in the spectral function due to the coupling of vector mesons to nucleon resonances have been predicted [5, 6]. Recently many studies have focused on the $\eta^{\prime}$ meson. Its

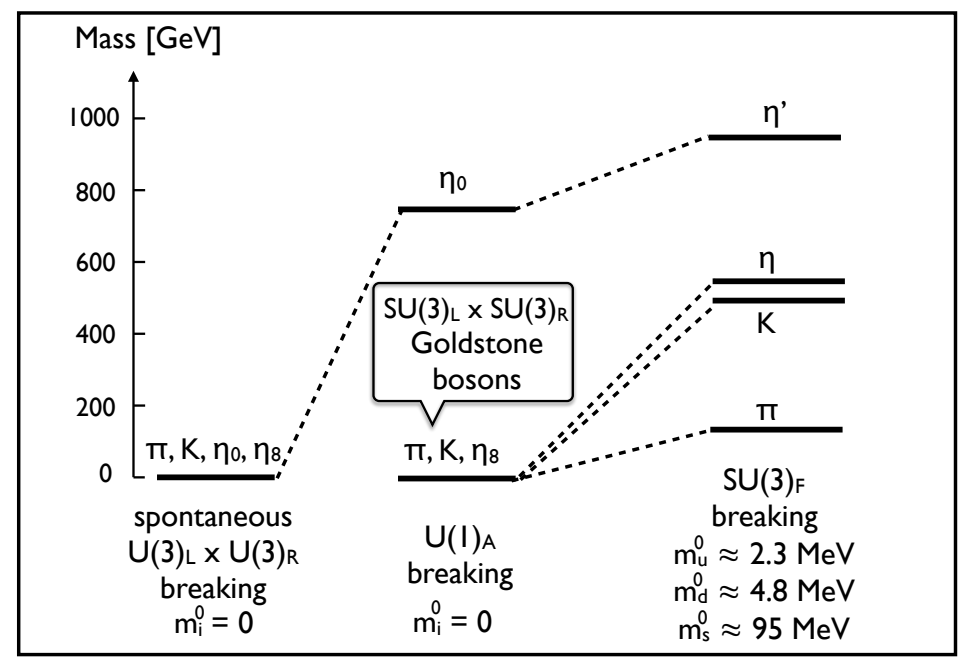

Figure 1: Symmetry breaking pattern for pseudoscalar mesons as described in the text, adapted from [7].

especially large mass compared to the mass of the other pseudoscalar mesons has to be attributed to chiral and flavor symmetry breaking effects. Pseudoscalar mesons are particularly suited for studying in-medium modifications, as shown in Fig. 1 [7]. Spontaneous chiral symmetry breaking generates a pseudo scalar nonet $\left(\pi, K, \bar{K}, \eta_{0}, \eta_{8}\right)$ of massless Goldstone bosons. The explicit breaking of the $\mathrm{U}(1)_{A}$ symmetry selectively shifts up the $\eta_{0}$-singlet mass, leaving the $\mathrm{SU}(3)$ flavour octet of pions, kaons and $\eta_{8}$ massless. Explicit chiral symmetry breaking by introducing non-zero quark masses then leads to the experimentally observed meson masses $[1,7,8]$. Since symmetry breaking has such a big effect on meson masses one would expect corresponding effects in case of a partial restoration of this symmetry in a strongly interacting medium.

Due to a reduction of the chiral condensate a drop in the $\mathrm{U}_{A}(1)$ breaking part of the $\eta^{\prime}$ mass might be expected $[9,10]$, causing an $\eta^{\prime}$ mass shift of $\approx-120 \mathrm{MeV}$ at nuclear matter density $\rho_{0}$. This prediction is, however, in conflict with earlier calculations within the Nambu-Jona-Lasiniomodel which expect almost no change in the $\eta^{\prime}$ mass as a function of nuclear density [11]. Further model calculations claim mass shifts of the $\eta^{\prime}$ of -80 [12] and $-40 \mathrm{MeV}$ [13] at $\rho_{0}$. It is obvious that these contradictory theoretical predictions call for an experimental clarification. 


\section{Experimental approaches to study mesons in the medium}

For long-lived mesons such as pions or kaons, meson beams can be used to study the mesonnucleus interaction experimentally. This is not possible for short-lived mesons like $\eta, \omega$, and $\eta^{\prime}$ mesons where such beams are not available. Here, one has to produce the mesons in a nuclear reaction and to study their interaction with nucleons or nuclei in the final state.

The meson-nucleus interaction and the in-medium modifications of mesons can be described by a complex potential:

$$
U(r)=V(r)+i W(r),
$$

where $\mathrm{V}(\mathrm{r})$ and $\mathrm{W}(\mathrm{r})$ represents the real and the imaginary part, respectively; $r$ is the distance between the meson and the centre of the nucleus [9]. The strength of the real part of the mesonnucleus potential is connected to the meson in-medium mass shift $\Delta m\left(\rho_{0}\right)$ at saturation density $\rho_{0}$ by:

$$
V(r)=\Delta m\left(\rho_{0}\right) \cdot c^{2} \cdot \frac{\rho(r)}{\rho_{0}} .
$$

The imaginary part of the potential describes the meson absorption in the medium via inelastic channels and is related to the in-medium width $\Gamma_{0}$ of the meson at nuclear saturation density by [14]

$$
W(r)=-\frac{1}{2} \Gamma_{0} \cdot \frac{\rho(r)}{\rho_{0}} .
$$

The imaginary part of the meson-nucleus potential corresponds to half of the in-medium width: $\operatorname{Im} U=\Gamma / 2$, which can be extracted from the attenuation of the meson flux deduced from a measurement of the so-called transparency ratio for a number of nuclei [15]. The transparency ratio describes the loss of flux of mesons in nuclei via inelastic processes. Due to these processes the lifetime of mesons in a nuclear medium is reduced leading to an increase in width as compared to the free particle width.

Weil et al. [16] discussed the possibility to extract information on the in-medium meson mass and the real part of the meson-nucleus potential from a measurement of the excitation function and/or momentum distribution of mesons in the photo production off a nucleus. Due to a lowering of the meson mass in the medium the meson production threshold will decrease and the enlarged phase space will consequently lead to an increase of the production cross section for a given incident beam energy as compared to a scenario without mass shift. The dropping of the meson mass in the medium also affects the momentum distribution of the produced meson in the final state. When a meson is produced with a lower mass, then its total energy is on average also reduced due to kinematics. In addition, mesons produced within the nuclear medium must regain their free mass upon leaving the nucleus. Thus, in case of an in-medium mass drop, this mass difference has to be compensated at the expense of their kinetic energy. GiBUU transport-model calculations [16] demonstrated that there will be a downward shift in the momentum distribution for near-threshold energies as compared to a scenario without mass shift. A mass shift can thus be indirectly inferred from a measurement of the excitation function as well as from the momentum distribution of the meson. This idea, initially worked out for $\omega$ mesons [16], has independently been pursued on a 
quantitative level for $\eta^{\prime}$ mesons by E. Paryev [17].

A necessary condition for the experimental observation of meson-nucleus bound states is that $|V|>>|W|$. It is therefore important to study experimentally the relative strengths of the real and imaginary part of the meson-nucleus interaction for the meson of interest. Then, by the observation of meson-nucleus bound states information about the real and imaginary part of the meson-nucleus optical potential can be gained.

The data on $\omega$ and $\eta^{\prime}$ photo production were taken with the Crystal Barrel/TAPS detector system at the ELSA accelerator in Bonn $[18,19,20]$. The high segmentation and the almost full solid angle coverage of the detector systems allowed the reconstruction of $\omega$ and $\eta^{\prime}$ mesons from multiphoton final states by invariant mass analysis. The decay modes used in the analyses were $\omega \rightarrow \pi^{0} \gamma \rightarrow 3 \gamma$ and $\eta^{\prime} \rightarrow \pi^{0} \pi^{0} \eta \rightarrow 6 \gamma$ with branching ratios of $8.2 \%$ and $8.5 \%$, respectively [21].

\subsection{Experimental results on the imaginary part of the $\omega$ - and $\eta^{\prime}$-nucleus potential}

To determine the meson attenuation in nuclei and the inelastic meson-nucleon cross sections as a function of the meson momentum, the transparency ratio is deduced from the data as defined in $[14,19,22]$. To suppress nuclear effects not related to meson absorption, the transparency ratio
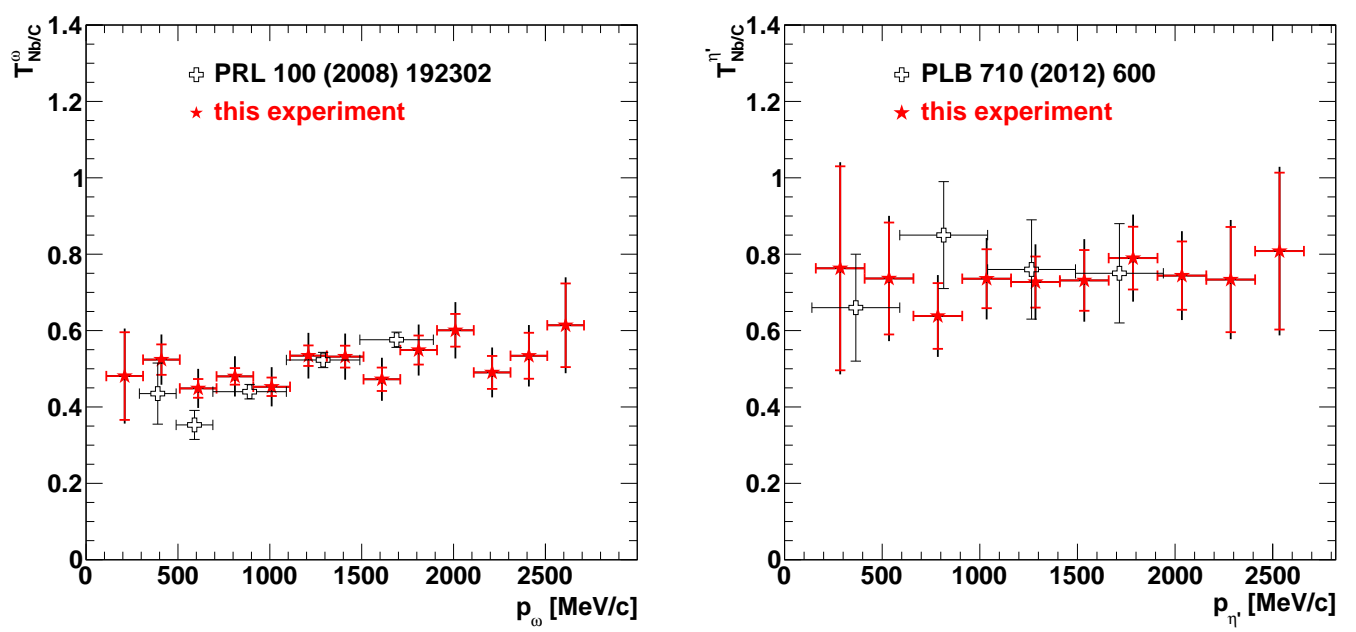

Figure 2: The transparency ratio according to Eq. 2.4 for (Left) $\omega$ and (Right) $\eta^{\prime}$ photoproduction off carbon and niobium (red stars) in comparison to earlier measurements (open crosses) [14, 22]. For the present data the thick error bars (red) represent the statistical errors. The thin error bars (black) include the systematic errors added in quadrature (the figure is taken from [19]).

is not taken relative to the free nucleon but rather to a nucleon in a light nucleus like carbon. The momentum dependence of the transparency ratio for a meson $m$ is thus obtained by dividing the differential inclusive meson production cross sections for e.g. niobium by the one for carbon, according to Eq.2.4

$$
T_{N b / C}^{m}\left(p_{m}\right)=\frac{12 \cdot \frac{d \sigma_{\gamma N b \rightarrow m X}}{d p_{m}}\left(p_{m}\right)}{93 \cdot \frac{d \sigma_{\gamma C \rightarrow m X}}{d p_{m}}\left(p_{m}\right)},
$$


where 12 and 93 are the nuclear mass numbers of carbon and niobium, respectively. The resulting transparency ratios as a function of the meson momentum are shown in Fig. 2.

A slight increase with momentum is observed for the $\omega$ meson while for the $\eta^{\prime}$ meson the transparency ratio is almost independent of momentum. Within the errors the results are consistent with earlier measurements $[14,22]$. The momentum dependence of the transparency ratio allows the determination of the in-medium $\omega$ and $\eta^{\prime}$ widths which can be converted into the dependence of the imaginary part of the $\omega$ - and $\eta^{\prime}$-nucleus potential as a function of the available energy in the meson $-{ }^{93} \mathrm{Nb}$ system, as shown in Fig. 3 (for more details see [19]). The data have been fitted and extrapolated towards the production threshold. For the $\omega$ meson the modulus of the imaginary part of the meson nucleus potential near threshold is found to be (48 \pm 12 (stat) \pm 9 (syst)) MeV. For the $\eta^{\prime}$ meson the extrapolation towards the production threshold yields an imaginary potential of -(13 \pm 3 (stat) \pm 3 (syst)) MeV.
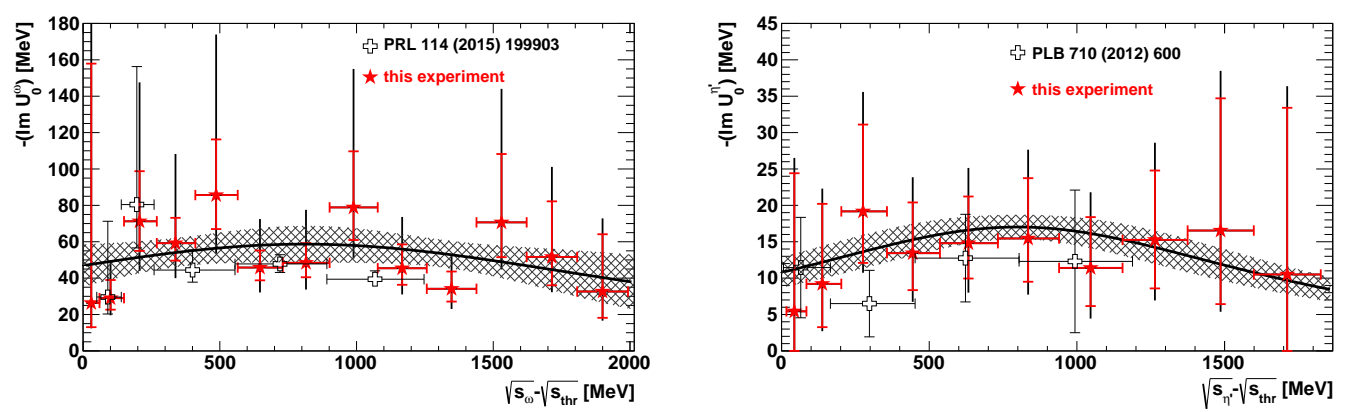

Figure 3: Imaginary part of the (Left) $\omega$-nucleus and (Right) $\eta^{\prime}$-nucleus optical potential as a function of the available energy in the meson- ${ }^{93} \mathrm{Nb}$ system (red stars) [19] in comparison to earlier measurements (open crosses) $[14,22]$. The solid curves are Breit-Wigner fits to the present data. The shaded areas indicate a confidence level of $\pm 1 \sigma$ of the fit curve taking statistical and systematic errors into account (the figure is taken from [19]).

\subsection{Experimental data on the real part of the $\omega$ - and $\eta^{\prime}$-nucleus potential}

Information on the in-medium meson mass and the real part of the meson-nucleus potential can be extracted from the excitation function of the meson near the production threshold [16, 17]. This method is sensitive to the nuclear density at the production point and hence it is applicable for all mesons, irrespective of their lifetime.

The experimental results for the $\omega$ excitation function in comparison to the GiBUU transport model [23] for 6 different scenarios allowing for mass shifts up to $-125 \mathrm{MeV}$ at normal nuclear density [24] excluded a strong mass shift scenario, $|V|>100 \mathrm{MeV}$ as predicted in [2, 4]. A $\chi^{2}$-fit of the data with the calculated excitation functions for the different scenarios gives a potential depth of $\mathrm{V}_{\omega A}\left(\rho=\rho_{0}\right)=-(42 \pm 17$ (stat) \pm 20 (syst) $) \mathrm{MeV}[24,25]$.

Experimental data for the $\eta^{\prime}$ excitation function in comparison to theoretical calculations are shown in Fig. 4 [18, 20]. In order to study the possible dependence of the real part of the $\eta^{\prime}$-nucleus optical potential on the mass of the nucleus, the data were taken on ${ }^{12} \mathrm{C}$ and ${ }^{93} \mathrm{Nb}$ targets with the CBELSA/TAPS detector system. In both cases the data disfavour a deep real potential of $|V|>100$ 

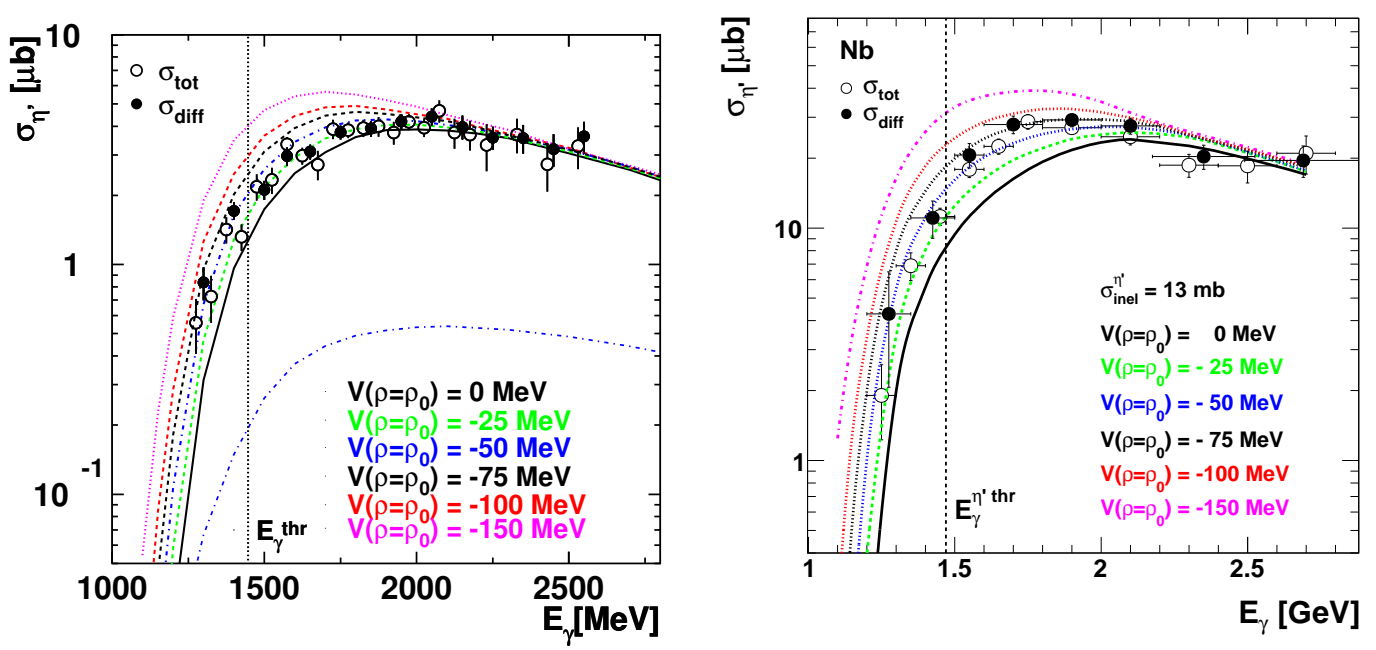

Figure 4: Measured excitation function for $\eta^{\prime}$ meson off ${ }^{12} \mathrm{C}$ (left) [18] and ${ }^{93} \mathrm{Nb}$ (right) [20], in comparison to theoretical calculations for different scenarios. The experimental data are extracted by integrating the differential cross sections (full circles) and by direct measurement of the $\eta^{\prime}$ yield in incident photon energy bins (open circles). The calculations are for $\sigma_{\eta^{\prime} N}=11 \mathrm{mb}$ (for $\mathrm{C}$ data) and for $\sigma_{\eta^{\prime} N}=13 \mathrm{mb}$ (for $\mathrm{Nb}$ data), and for potential depths: $V=0 \mathrm{MeV}$ (black line), $-25 \mathrm{MeV}$ (green), $-50 \mathrm{MeV}$ (blue), $-75 \mathrm{MeV}$ (black dashed), $-100 \mathrm{MeV}$ (red) and $-150 \mathrm{MeV}$ (magenta) at normal nuclear density, respectively, and using the full nucleon spectral function. The dot-dashed blue curve (in the left spectrum) is calculated for correlated intranuclear nucleons only. The figures are taken from [18, 20].

$\mathrm{MeV}$, as predicted in [9]. The calculations have been normalized to the experimental data for incident photon energies of $\mathrm{E}_{\gamma}>2.2 \mathrm{GeV}$. This normalization is within the systematic error of the cross section data (23\%) and the calculations (10-15\%). The highest sensitivity to the $\eta^{\prime}$ potential depth is found for incident photon energies near and below the production threshold on the free nucleon. The excitation function data on both nuclei appear to be incompatible with $\eta^{\prime}$ mass shifts of -100 $\mathrm{MeV}$ and more at normal nuclear matter density, as clearly seen in Fig. 4. A $\chi^{2}$-fit of the data with the calculated excitation functions for the different scenarios over the full range of incident energies gives a potential depth of $-(40 \pm 6) \mathrm{MeV}$ for $\eta^{\prime}-\mathrm{C}$ [18] and $-(40 \pm 12) \mathrm{MeV}$ for $\eta^{\prime}-\mathrm{Nb}$ [20].

It has been investigated whether the observed cross section enhancement relative to the $V=0 \mathrm{MeV}$ case could also be due to $\eta^{\prime}$ production on dynamically formed compact nucleonic configurations - in particular, on pairs of correlated nucleon clusters - which share energy and momentum. These effects have been studied experimentally [26] and theoretically [27, 28] in very near-threshold $K^{+}$ production in proton-nucleus reactions. Applying the parametrization of the spectral function given by [29], in Fig. 4 (left: dot-dashed blue curve) has been shown that correlated high momentum nucleons contribute only about $10-15 \%$ to the $\eta^{\prime}$ yield in the incident energy regime above 1250 $\mathrm{MeV}$ [18]. The observed cross section enhancement can therefore be attributed mainly to the lowering of the $\eta^{\prime}$ mass in the nuclear medium.

The measured momentum differential cross sections for $\eta^{\prime}$ meson photoproduction off $\mathrm{C}[18]$ and $\mathrm{Nb}$ [20] are shown in Fig. 5. The average momentum in both cases is $\approx 1.1 \mathrm{GeV} / c$. Bin sizes of 0.1 $\mathrm{GeV} / c$ for $\mathrm{C}$ data and $\geq 0.2 \mathrm{GeV} / c$ for $\mathrm{Nb}$ data have been chosen which are large compared to the 
momentum resolution of $25-50 \mathrm{MeV} / c$ deduced from the experimental energy resolution and from Monte Carlo simulations. The $\eta^{\prime}$ momentum distributions have been calculated for the incident photon energy range 1.3-2.6 GeV and for different potential depths $\mathrm{V}=0,-25,-50,-75,-100$ and $-150 \mathrm{MeV}$. The comparison of these calculations with the data again excludes strong $\eta^{\prime}$ mass shifts. A $\chi^{2}$-fit of the data with the calculated momentum distributions for the different scenarios
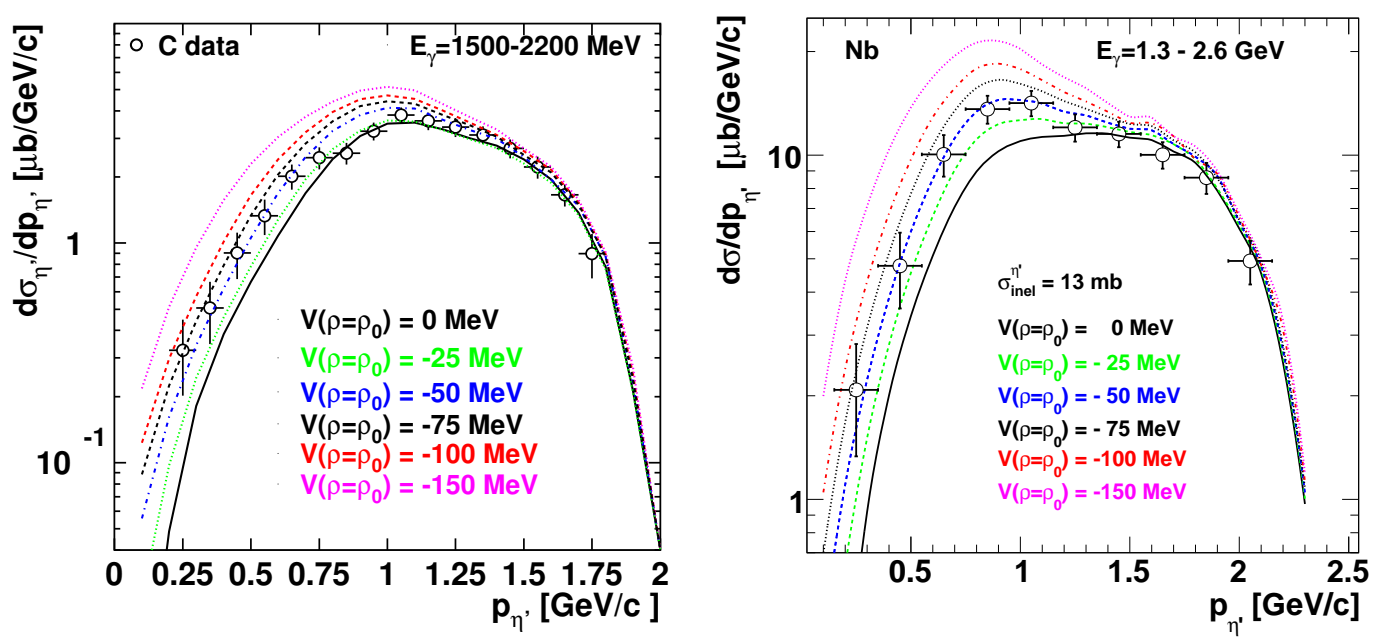

Figure 5: Left: Momentum distribution for $\eta^{\prime}$ photoproduction off $\mathrm{C}$ for the incident photon energy range $1500-2200 \mathrm{MeV}$. The calculations are for $\sigma_{\eta^{\prime} N}=11 \mathrm{mb}$ and have been reduced by a factor 0.75 (see text) [18]. Right: Momentum distribution for $\eta^{\prime}$ photoproduction off $\mathrm{Nb}$ for the incident photon energy range 1.3-2.6 $\mathrm{GeV}$. In both spectra the theoretical curves are for potential depths $\mathrm{V}=0,-25,-50,-75,-100$ and -150 $\mathrm{MeV}$ at normal nuclear density. The color code is identical to the one in Fig. 4. The figures are taken from $[18,20]$.

over the full range of incident energies gives a potential depth of $-(32 \pm 11) \mathrm{MeV}$ for $\mathrm{C}[18]$ and $-(45 \pm 20) \mathrm{MeV}$ for $\mathrm{Nb}[20]$.

To study the possible momentum dependence of the real part of the meson-nucleus optical potential an experiment has been performed to produce mesons with low momenta $(<500 \mathrm{MeV} / c)$. In the reaction $\gamma C \rightarrow \omega\left(\eta^{\prime}\right) p \rightarrow \pi^{0} \gamma\left(\pi^{0} \pi^{0} \eta\right) p$ the proton has been measured in the TAPS detector covering the polar angle range $\Theta_{p}=1^{\circ}-11^{\circ}$. In this case the forward going proton takes over most of the momentum of the incoming photon beam. Being so low in energy, the $\omega\left(\eta^{\prime}\right)$ mesons are particularly sensitive to the meson-nucleus potential. In case of a strong repulsive (attractive) interaction one would expect the peak in the kinetic energy distribution to be shifted to higher (lower) energies for the $\mathrm{C}$ target. The sensitivity of the peak position in the kinetic energy distribution on the potential depth, studied in [30], can be exploited to deduce the depth of the real part of the $\omega$-nucleus potential. The correlation between the potential depth and the peak in the kinetic energy distribution compared with the experimentally determined peak position indicates a potential depth of $-(15 \pm 35) \mathrm{MeV}$ [30]. The same approach has been applied for the $\eta^{\prime}$ meson to deduce the real part of the $\eta^{\prime}$-nucleus potential at average momenta of $\eta^{\prime}$-mesons $\approx 600 \mathrm{MeV} / \mathrm{c}$. The analysis is still ongoing. 
Combining the results from the analysis of the excitation function and the peak position of the kinetic energy distribution an average depth of the real part of the $\omega$-C potential of $\mathrm{V}\left(\rho=\rho_{0}\right)=$ $-(29 \pm 19$ (stat) \pm 20 (syst)) MeV is obtained Fig. 6 (left).

From the analysis of the excitation functions and the momentum distributions and by proper
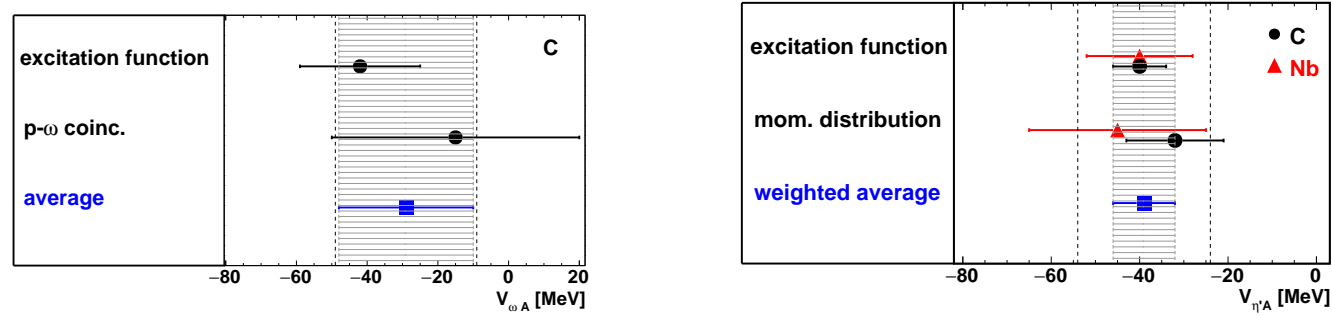

Figure 6: Depths of the real part of the $\omega$ (left) and $\eta^{\prime}$ (right) -nucleus potential determined by analyzing the excitation function and the momentum distributions for $\mathrm{C}[18,24]$ (full black circles) and for $\mathrm{Nb}$ [20] (red triangles). The weighted overall average is indicated by a blue square and the shaded area. The vertical hatched lines mark the range of systematic uncertainties. The figures are taken from [31].

weighting of the errors a depth of the real part of the $\eta^{\prime}-\mathrm{C}$ and $-\mathrm{Nb}$ potential of $\mathrm{V}\left(\rho=\rho_{0}\right)=-(39$ \pm 7 (stat) \pm 15 (syst)) $\mathrm{MeV}$ is obtained (see Fig. 6 (right)). The systematic error quoted is mainly due to uncertainties in normalizing the calculations to the data.

This result for $\mathrm{V}\left(\rho=\rho_{0}\right)$ is consistent with predictions of the $\eta^{\prime}$-nucleus potential depth within the Quark-Meson Coupling model (QMC) [13] and with calculations in [32] but does not support larger mass shifts as discussed in $[9,12]$.

\section{Search for meson-nucleus bound states}

The interaction of neutral mesons with nuclei has been studied to find out whether mesonnucleus states might exist as well, only bound by the strong interaction. Here, the meson-nucleus interaction has to be sufficiently attractive and the meson absorption in nuclei should be relatively weak to allow the formation of relatively narrow states. Considering the results obtained for the $\omega$ and $\eta^{\prime}$-nucleus potentials it is evident that the $\eta^{\prime}$ meson is a much better candidate for the formation of meson-nucleus bound states than the $\omega$ meson since we found for the $\eta^{\prime}$ meson that $|W|<<|V|$, while the opposite was observed for the $\omega$ meson, as illustrated in Fig. 7. For the $\eta^{\prime}$ meson there is a possibility for the existence of relatively narrow bound states while for the $\omega$ meson the imaginary potential is comparable to the real one and only broad structures can be expected which makes it difficult to detect them experimentally.

Since the $\eta^{\prime}$ meson is the better candidate for the search of mesic states several corresponding experiments have been launched. At the Fragment Separator (FRS) at GSI an experiment to search for $\eta^{\prime}$ bound states has been performed via missing mass spectroscopy in the ${ }^{12} \mathrm{C}(\mathrm{p}, \mathrm{d})$ reaction in almost recoil free kinematics [33]. The results recently published in [34] conclude that a strongly attractive potential of $V_{0} \approx-150 \mathrm{MeV}$ predicted by the NJL model [9] is rejected for a relatively shallow imaginary potential [19]. An alternative approach is the photoproduction of $\eta^{\prime}$ mesons in the ${ }^{12} \mathrm{C}(\gamma, \mathrm{p})$ reaction, again in almost recoil-free kinematics. In the ongoing experiment at 


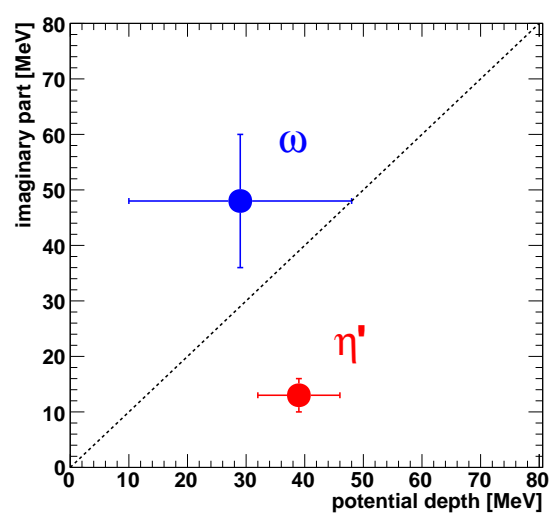

Figure 7: The imaginary part versus the real part of the meson-nucleus potential for $\omega$ and $\eta^{\prime}$ mesons. The figures are taken from [31].

the LEPS2 facility (Spring8) [35] the missing mass spectroscopy is combined with detecting the decay of the $\eta^{\prime}$ mesic state in a semi-exclusive measurement. An analogous photoproduction measurement has been proposed for the BGO-OD setup at the ELSA accelerator in Bonn [36]. A semi-exclusive measurement has also been considered for the Super-FRS at FAIR [37].

\section{Conclusion}

The photoproduction of $\omega$ and $\eta^{\prime}$ mesons off nuclei has been studied in the 1-3 GeV energy range to extract information on the in-medium properties of these mesons. The interaction of mesons with nuclei and the in-medium effects have been described with a complex potential, comprising a real part, associated with the mass modification in the nuclear medium, and an imaginary part, accounting for the absorption of mesons in nuclei. For the $\omega$ - and $\eta^{\prime}$ - nucleus potential values of $\mathrm{U}_{\omega A}\left(\rho=\rho_{0}\right)=-((29 \pm 19$ (stat) \pm 20 (syst) $)+\mathrm{i}(48 \pm 12$ (stat) \pm 9 (syst) $)) \mathrm{MeV}$ and $\mathrm{U}_{\eta^{\prime} A}\left(\rho=\rho_{0}\right)$ $=-((39 \pm 7$ (stat) \pm 15 (syst) $)+\mathrm{i}(13 \pm 3$ (stat) \pm 3 (syst) $)) \mathrm{MeV}$ have been obtained. The latter measurement establishes for the first time an in-medium mass drop of a pseudoscalar meson at normal nuclear matter density. Although not very deep, the real part of the $\eta^{\prime}$ - nucleus potential is about three times larger than the imaginary part and may thus allow the existence and observation of $\eta^{\prime}$-nucleus states, only bound by the strong interaction. Corresponding experiments are ongoing.

\section{References}

[1] V. Bernard and U.-G. Meissner, Nucl. Phys. A 489, 647 (1988)

[2] T. Hatsuda and S. Lee, Phys. Rev. C 46, R34 (1992)

[3] G. E. Brown and M. Rho, Phys. Rev. Lett. 66, 2720 (1991)

[4] F. Klingl et al., Nucl. Phys. A 610, 297 (1997), Nucl. Phys. A 650, 299 (1999)

[5] P. Mühlich et al., Nucl. Phys. A 780, 187 (2006) 
[6] M. Lutz et al., Nucl. Phys. A 706, 437 (2002)

[7] S. Klimt et al., Nucl. Phys. A 516, 429 (1990)

[8] V. Bernard, R. L. Jaffe and U.-G. Meissner, Nucl. Phys. B 308753 (1988)

[9] H. Nagahiro, M. Takizawa, and S. Hirenzaki, Phys. Rev. C 74, 045203 (2006)

[10] Y. Kwon et al., Phys. Rev. D 86, 034014 (2012)

[11] V. Bernard and U.-G. Meissner, Phys. Rev. D 38, 1551 (1988)

[12] S. Sakai and D. Jido, Phys. Rev. C 88, 064906 (2013)

[13] S. D. Bass and A. W. Thomas, Phys. Lett. B 634368 (2006)

[14] M. Nanova et al., CBELSA/TAPS Collaboration, Phys. Lett. B 710, 600 (2012)

[15] D. Cabrera et al., Nucl. Phys. A 733, 130 (2004)

[16] J. Weil, U. Mosel, and V. Metag, Phys. Lett. B 723, 120 (2013)

[17] E. Ya. Paryev, J. Phys. G: Nucl. Part. Phys. 40, 025201 (2013)

[18] M. Nanova et al., CBELSA/TAPS Collaboration, Phys. Lett. B 727, 417 (2013)

[19] S. Friedrich et al., CBELSA/TAPS Collaboration, Eur. Phys. J. A 52, 297 (2016)

[20] M. Nanova et al., CBELSA/TAPS Collaboration, Phys. Rev. C 94, 025205 (2016)

[21] K. A. Olive et al., Particle Data Group, Chin. Phys. C 38, 090001 (2014)

[22] M. Kotulla et al., CBELSA/TAPS Collaboration, Phys. Rev. Lett. 100, 192302 (2008), Phys. Rev. Lett. 114, 199903 (2015)

[23] O. Buss et al., Phys. Rep. 512, 1 (2012)

[24] V. Metag et al., A2 Collaboration, Prog. Part. Nucl. Phys. 67, 530 (2012)

[25] V. Metag, Hyp. Int. 234, 25 (2015)

[26] M. Debowski et al., Acta Phys. Polonica B 27, 3015 (1996)

[27] A. Sibirtsev, W. Cassing, and U. Mosel, Z. Phys. A 358, 357 (1997)

[28] E. Ya. Paryev, Eur. Phys. J. A 5, 307 (1999)

[29] S. V. Efremov and E. Ya. Paryev, Eur. Phys. J. A 1, 99 (1998)

[30] S. Friedrich et al., CBELSA/TAPS Collaboration, Phys. Lett. B 736, 26 (2014)

[31] M. Nanova and V. Metag, EPJ Web Conf 130, 02007 (2016)

[32] H. Nagahiro, S. Hirenzaki, E. Oset, and A. Ramos, Phys. Lett. B 709, 87 (2012)

[33] K. Itahashi et al., Prog. Theo. Phys. 128, 601 (2012)

[34] Y. K. Tanaka et al., Phys. Rev. Lett. 117, 202501 (2016)

[35] N. Muramatsu et al., arXiv:1307.6411

[36] V. Metag et al., approved proposal ELSA/3-2012-BGO

[37] H. Nagahiro, D. Jido, H. Fujioka, K. Itahashi, and S. Hirenzaki, Phys. Rev. C 87, 045201 (2013) 